\title{
Analysis and Suggestions on Measurement Error of Electric Energy Meter
}

\author{
Guo Shujiang', Jiang Ling ${ }^{2}$, Xu Jianchun ${ }^{2}$, Su Hainan ${ }^{2}$, Guo Deyi ${ }^{1}$, Chen Defang ${ }^{1}$, Yue \\ Yangzhuo ${ }^{1}$, Shi Guodong ${ }^{1}$, Tao Shuyi ${ }^{1}$, Liu Bo ${ }^{1}$ \\ 1. Fushun Power Supply Company, Liaoning Electric Power Company Limited, State Grid, China \\ 2. Dandong Power Supply Company, Liaoning Electric Power Company Limited, State Grid, China.
}

Keywords: electric energy meter; measurement error; cause analysis; standard current

\begin{abstract}
With the transformation of power network in recent years, the metering function of electric energy meters plays an important role in electric power work. Due to the defects of the electric energy meter and the external uncertainties, measurement error is easy to cause when measured. In addition, a certain amount of measurement error in the metering process of electric energy meters will be caused by voltage instability, which will cause great trouble to electric workers, and they need to be paid enough attention to. This paper analyzes the causes of the measurement error of the electric energy meter, discovers existing problems, and proposes effective and reasonable suggestions for reducing measurement errors.
\end{abstract}

\section{Introduction}

The electric energy meter is a kind of multi-function instrument that can not only be used to measure electric energy. According to the working principle and structure of the electric energy meter, it can be divided into three categories, electromechanical integration, electronic and inductive. Due to the defects of the electric energy meter and the external uncertainties, the measurement error is easy to cause when measured. The error caused by the measurement data will seriously damage the interests of both the power supply enterprise and the users. The accuracy of electric energy meter measurement is conducive to ensuring fairness between users and power supply enterprises. Therefore, it is of great significance to promote the development of electric energy meters by analyzing the error of the meter, finding out the reason and offering suggestions.

\section{Cause of Measurement Error of Electric Energy Meter}

\subsection{Internal causes related to measurement error of electric energy meter}

\subsubsection{The influence of voltage, current and temperature on measurement error}

The rotary pulley of the electric energy meter will change, when the voltage of the inner line of the electric energy meter is different from the external line. The instability of this change will affect the metering of the electric energy meter and make it produce measurement error. Therefore, it is very difficult to measure the energy meter accurately, when the voltage is unstable. In the same way, if the current in the meter is not the same as the current in the external and inner line, it will cause a certain deviation in the meter when measuring, which makes the electricity consumption displayed by the meter not match the actual electricity consumption. According to Joule's law, when the current passes through the electric energy meter, a certain amount of heat is generated, thus increasing the temperature in the electric energy meter. So the resistivity of this resistance will be changed. From the influence of voltage and current transmission, additional temperature errors will be generated in the electric energy meter.

\subsubsection{The influence of voltage on measurement error in electric energy meter line.}

The influence of voltage on measurement error in electric energy meter line is mainly caused by voltage asymmetry. First of all, there are great differences in the accessories inside the meter. When the meter is placed in the same circuit, the voltage and current will make the accessories of the 
meter form an unbalanced state, which will lead to the change of the pulley. The second is that even when the power meter's inner accessories are exactly the same, a relatively stable situation is produced, but if the voltage is asymmetrical at this time, the rotating pulley will change, the final absolute value is different, and the meter still has the error of measurement error. Finally, on the same line, the asymmetrical voltage makes the voltage control torque and the rotating pulley torque negatively correlated, so that the additional error is generated.

\subsection{External factors related to measurement errors of electric energy meters}

\subsubsection{Error in calculation}

In the calculation of electric energy meters, the calculation formula is a factor that cannot be neglected in the cause of error. High precision $\mathrm{B} / \mathrm{C}$ is used in the electric energy meter which is widely used at present to transform digitally. Then it is calculated by the energy metering formula of the electric energy meter. However, the error caused by the mode except the high precision $\mathrm{B} / \mathrm{C}$ is often ignored and is not taken into account. High precision $\mathrm{B} / \mathrm{C}$ computing is usually 6 digit calculation. Because of the complexity of calculation, the accuracy rate is low, so the calculation error caused by the calculation formula should be taken into account.

\subsubsection{Position tilt of the electric energy meter installation position}

The tilt of the installation position of the electric energy meter is also an external factor that cannot be neglected. When the electric energy meter is running normally, the electric energy meter can be easily shocked by the collision of the electric power workers, which makes the position of the instrument inclined, and eventually makes the meter error when measuring. The main reason is that the devices inside the electric energy meter are unreliable, not connected to a whole, as long as a little collision will make the elements inside the electric energy meter shift, and the torsional torque of the rotating pulley changes. Another reason is that the electric energy meter industry standard stipulates that the maximum measurement error is that when the current is less than $40 \%$ of the standard current, the torque of the rotating pulley of the electric energy meter is minimal, and the measurement error caused by the position tilt can be ignored, and it is not calculated. The position error of the electric energy meter is essentially similar to that of the rotary disk displacement error which causes greater tilt angle_- the greater the dip angle, the greater the lateral pressure and the inclination error. In general, the correct choice of the electric energy meter element and the rotating pulley and placing them in the center of the electric energy meter can reduce the displacement in the bearing caused by the rotating pulley, thus reducing the measurement error caused by the position tilt.

\section{Suggestions on Improvement of Measurement Error of Electric Energy Meter}

When the meter error is corrected, the first step should be to carefully check the performance of the electric energy meter and whether it can operate normally. Of course, there are many other ways to check. For example, it can be seen by direct observation of the electric energy meter, or whether the running can be executed from the test, and can also be tested for the rotating pulley and the power meter. There are many requirements for implementation of these methods. The most important responsibility for electric workers is to see the performance of the meter and ensure its normal operation. In addition, if some other indicators in the meter are not up to standard, the metering error of the energy meter is likely to be caused. The technical indicators of these indicators are the most important. If they do not meet the targets, the electric power meter will probably be paralyzed and unable to function. To sum up, the meter error correction is made to meet the requirements of the national verification procedures, so that the user's capital is saved.

A relatively low load electric energy meter should be used to measure and reduce the possibility of such errors. A low load electric energy meter can be used to calculate the amount of electricity consumed by the power users and the amount of harmonics absorbed. Finally, the total energy consumed by the user is calculated together. In addition, the load power can also be referred to. 
When the load power is greater than 0 , too much energy meter data is calculated, and it absorbs harmonics. When the load power is less than 0, energy meter is too small to transmit harmonic when it is calculated. According to the above two methods, it can be determined that the meter is too much or too small, and the final fees will be adjusted according to these phenomena. The size and direction of the load cannot be judged, so the amount of the load absorbed from the power grid and the harmonic generated from the power grid cannot be determined, thus the data of the meter cannot truly reflect the influence of the nonlinear load on the power grid.

In addition, a reasonable metering location can be selected as close to the scene as possible to reduce the loss of electric energy in the secondary circuit and load. It is necessary to choose reasonable electric energy meter and mutual inductor to compensate, reduce the error caused by the metering device, and prepare for every test before putting into production. In the future operation and management process, regular inspection and rotation system should be carried out according to the current standards.

\section{Conclusion}

To sum up, the cause analysis of measurement error of electric energy meters can be divided into internal cause analysis and external cause analysis. In the specific revision process, appropriate measures shall be taken to make amendments according to the specific circumstances. The measurement error of the electric energy meter is analyzed and proposed, which is beneficial to the efficiency of the electric energy meter, and the interests of the power supply enterprises and the users are guaranteed.

\section{References}

[1] Zhang Jinfu, Zou Qin. Discussion on Reducing the Comprehensive Error of Electric Energy Metering Device [J]. Journal of Jiangxi Vocational and Technical College of Electricity.2008 (22).

[2] Wang Jiancai, Chen Aina. How to Improve the Accuracy of Electric Energy Measurement [J]. Rural Electrification.2010 (12).

[3] Huang Ting, Wang Guoyou. Analysis of Interference Factors and Solutions Affecting the Accurate Measurement of Electric Energy Meter [J]. Jiangsu Present Day Metrology. 2009 (12). 